\title{
Predictive Role of Large Unstained Cells (LUC) and Hematological Data in the Differential Diagnosis of Orchitis and Testicular Torsion
}

\author{
Bugra Bilge Keseroglu ${ }^{1(\mathbb{D})}$, Bulent Gungorer ${ }^{2(\mathbb{D})}$ \\ ${ }^{1}$ Department of Urology, Ankara City Hospital, Ankara, Turkey \\ ${ }^{2}$ Department of Emergency Medicine, Ankara City Hospital, Ankara, Turkey
}

Copyright@ Author(s) - Available online at https://dergipark.org.tr/en/pub/mbsjohs

Content of this journal is licensed under a Creative Commons Attribution-NonCommercial 4.0 International License,

Received: 18 February 2020, Accepted: 05 April 2021, Published online: 30 April 2021

(C) Ordu University Institute of Health Sciences, Turkey, 2021

\begin{abstract}
Objective: The main aim of this retrospective evaluation of patients presenting with the complaint of acute scrotal pain was to investigate whether large unstained cells (LUC) in the hematological data, percentage of LUC, and the other haematological parameters would be of benefit in differential diagnosis. The secondary aim was to investigate the utility of LUC and LUC\% as a new biochemical marker in the differential diagnosis of TT and EO.

Methods: In this study, a retrospective evaluation was made of patients who presented with the complaint of acute scrotal pain at the emergency polyclinic. The patients were evaluated in three groups; testicular torsion, orchitis, and the control group. Primarily the large unstained cell and other hematological data of the patients were evaluated.

Results: Statistical differences were evaluated in LUC, LUC\%, platelet, neutrophil, leucocyte, mean platelet volume, neutrophil / lymphocyte, platelet / lymphocyte values among the groups ( $\mathrm{p}$ values respectively 0.001 , $<0.001,0.491,<0.001,<0.001,0.031,<0.001,0.001)$. The cutoff values for the differentiation of epididimoorchitis (EO) were determined in the differential diagnosis of EO and TT. The highest area under curve values were found for LUC, LUC\% and neutrophils, respectively, 0.752 (0.660-0.843), 0.698 (0.605-0.790), $0.383(0.284-0.482)$.

Conclusion: The results of this study showed that the LUC and LUC\% values obtained from complete blood count (CBC) can be reliably used in the differential diagnosis of EO and TT
\end{abstract}

Key words: Orchitis, large unstained cell (LUC), testis torsion, predictive value, nlr

Suggested Citation: Keseroglu BB, Gungorer B.; Predictive role of large unstained cells (LUC) and hematological data in the differential diagnosis of orchitis and testicular torsion. Mid Blac Sea Journal of Health Science, 2021; 7(1):97-103

\section{Address for correspondence/reprints:}

Bugra Bilge Keseroglu,

Telephone number: +90 (312) 5526000

E-mail: bugrakes@gmail.com 


\section{Introduction}

Testis torsion (TT) is one of the most important and frequently seen emergency conditions in urology practice. In males aged 1-25 years, it is seen at an annual frequency of 4.5/100,000 (1). Testis torsion occurs as a result of the spermatic cord wrapping around the longitudinal axis, causing first a venous block then an arterial block. If intervention is made for TT within the first 6 hours, there is a $90 \%-100 \%$ chance of recovery without injury to the testis tissue, if treatment is applied in 6-12 hours this rate is $20 \%$ $50 \%$, and if at $12-24$ hours, $0 \%-10 \%$ (2). When TT is not treated as an emergency and appropriately, infertility and testis loss can result because of germinal cell damage. In the differential diagnosis, the disease with which TT is most confused is epididymo- orchitis (EO).

An important differentiating examination finding of EO is a decrease in pain with elevation of the scrotum (Prehn sign) (3). However, the same clinical findings have been shown in patients diagnosed with torsion (4). Power Doppler ultrasonography (DUS) is used most often in the differential diagnosis and this test has high reported rates of sensitivity (86-100\%) and specificity (95-100\%) (5). However, not all centres have the facilities for DUS of patients at the time of presentation at an emergency polyclinic. This is a great disadvantage for TT cases requiring emergency treatment and leads to the risk of unnecessary surgical treatment. Therefore, clinicians have been motivated to search for more easily accessible methods to facilitate the differential diagnosis. Parameters have been previously researched on this subject, such as several hematological parameters, leukocyte count, neutrophil/leukocyte ratio (NLR), and mean platelet volume (MPV) $(6,7)$.

The main aim of this retrospective evaluation of patients presenting with the complaint of acute scrotal pain was to investigate whether large unstained cells (LUC) in the hematological data, and the percentage of LUC, and the systemic anti-inflammatory response data (NLR, MPV) would be of benefit in differential diagnosis. The secondary aim was to investigate the utility of LUC and LUC\% as a new biochemical marker in the differential diagnosis of TT and EO.

\section{Methods}

\section{Study population}

In this study, a retrospective evaluation was made of patients who presented with the complaint of acute scrotal pain at the Emergency and Urology Polyclinic of Ankara City Hospital between March 2019 and
August 2020. The group evaluated ranged in age between 3 and 37 years. The protocol was approved by local Ethics Committee at Ankara City Hospital (Protocol No: E1-20-1126). The patients included in the study were those who were applied a scrotal Doppler ultrasonography, were then diagnosed with TT or EO and treated. Patients included in the TT diagnosis group had presented at the emergency polyclinic within 12 hours of the onset of pain and were treated with surgery (detorsion and orchiectomy). The EO patient group were also accepted as those with scrotal pain for a maximum of 12 hours, and an upper age limit of 37 years was determined. All of the orchitis patients participating in this study were unilateral.A control group was formed of healthy individuals with no similar complaints. Patients were excluded from the study if they had any liver, kidney or hematological disease, were receiving any treatment (chemotherapy, steroids) that could affect secondary hematological results, or had a history of scrotal surgery or scrotal trauma.

\section{Laboratory and radiologic analyses}

All the patients were examined by the emergency specialist and an urology specialist. Scrotal DUS was applied to all the patients and recorded. Venous blood samples were withdrawn into tubes containing ethylene-diamine-tetra-acetic acid (EDTA) for the measurement of hematological parameters of the patients and the control group. The blood samples were sent to the hospital's central laboratory, and as routine, were analyzed with the flow cytometry method on a Siemens Advia 2120i ${ }^{\circledR}$ device within 20 minutes of reaching the laboratory. All the hematological parameters were stated as $103 / \mu \mathrm{L}$. Using these parameters, the NLR and platelet/lymphocyte ratio (PLR) were calculated. The MPV value was denominated in femtoliter.

\section{Statistical analysis}

Data obtained in the study were analyzed statistically using SPSS for Windows vn 22.0 software (SPSS ${ }^{\circledR}$, Chicago, IL, USA). Group parameters were stated as mean \pm standard deviation (SD) values. Conformity of the data to normal distribution was assessed with the KolmogorovSmirnov test. In the comparison of groups not showing normal distribution, the non-parametric Kruskal-Wallis test was used and the Tamhane posthoc analysis was completed. A value of $p<0.05$ was accepted as statistically significant. Receiver Operating Characteristic (ROC) curve analysis was performed to determine the cutoff, sensitivity, 
specificity, positive predictive value (PPV) and negative predictive value (NPV) of the hematological data.

\section{Results}

A retrospective evaluation was made of the medical records of 129 male patients. The patients were separated into 3 groups, as the TT group (n:39) treated with orchiectomy or surgical detorsion and testicular fixation, the EO group (n:49), and a control group (n:41) of healthy males. The mean age was $17.9 \pm 6.7$ years in the TT group, $19.4 \pm 6.9$ years in the EO group, and $19.0 \pm 7.8$ years in the control group. No difference was determined between the groups in respect of mean age.

No statistically significant difference was determined between the TT and EO groups in respect of platelet count and MPV. A statistically significant difference was determined between the groups in respect of LUC, LUC\%, neutrophil count, lymphocyte count, leukocytes, NLR and PLR (Table 1). LUC distribution according to groups is shown in Figure 1 and LUC\% in Figure 2.

The cutoff values for the differentiation of EO were determined in the differential diagnosis of EO and TT. As the predictive values for leukocytes, MPV, and thrombocytes had no statistical significance, and were not calculated. The sensitivity and specificity values, NPV and PPV were determined for LUC, LUC\%, neutrophils, NLR and PLR (Table 2). A ROC curve was drawn for each parameter (Figure 3).

Table 1 Comparison of parameters according to groups

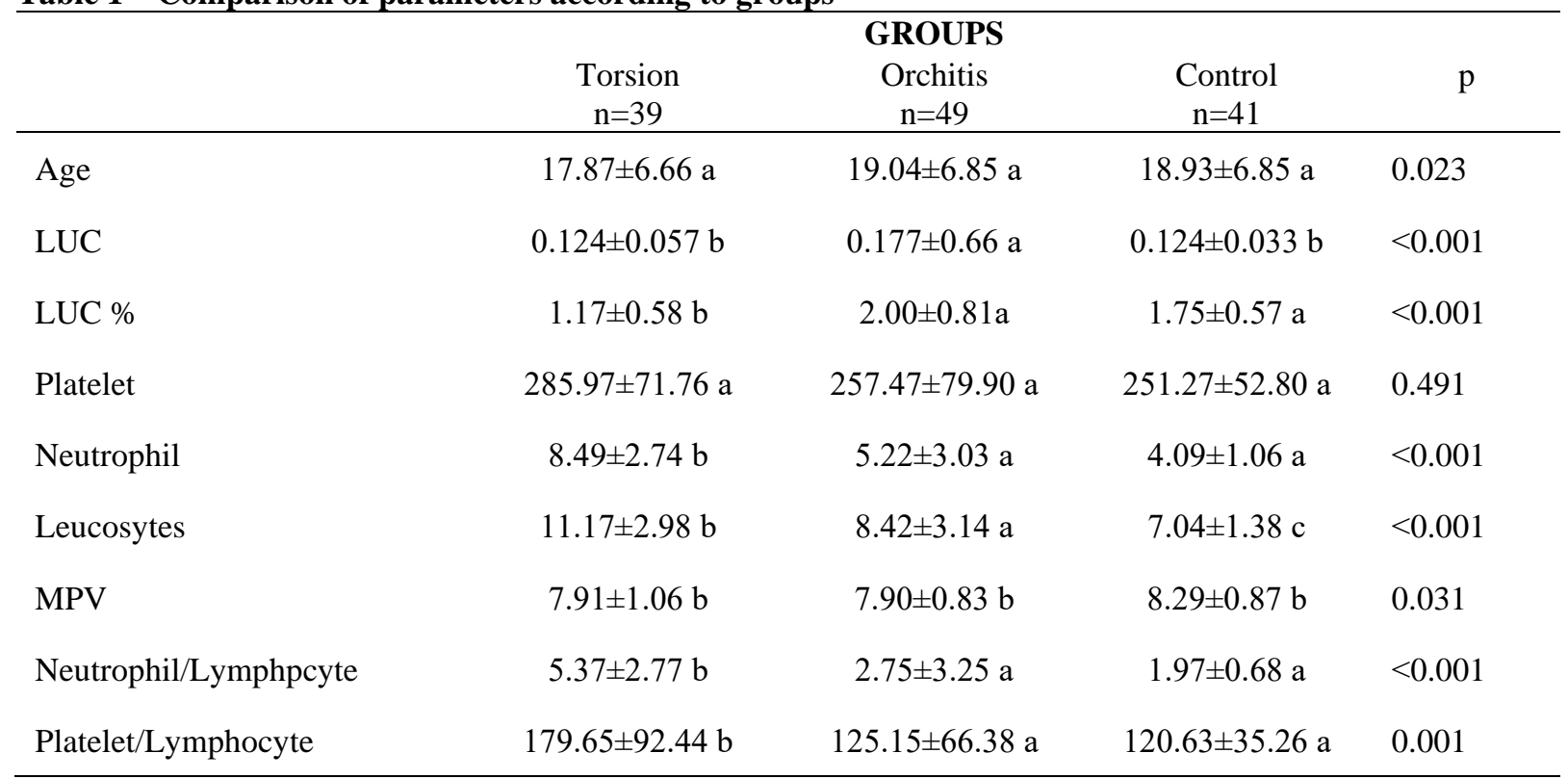

Arithmetic mean \pm standard deviation; $a, b$, c: no difference between groups with the same letter.LUC: Large Unstained cell, NLR:Neutrophil/Lymphocyt, PLR: Platelet/Lymphocyte

Table 2 Receiver operating characteristic analysis for the epididymitis group

\begin{tabular}{llcccccc}
\hline & AUC & Cut-off & $\mathrm{p}$ & Sensivity & Specificity & PPV & NPV \\
\hline LUC & $0.752(0.660-0.843)$ & 0.135 & 0.000 & 71.4 & 66.3 & 56.5 & 79.1 \\
LUC \% & $0.698(0.605-0.790$ & 1.55 & 0.000 & 71.4 & 78.8 & 67.3 & 81.8 \\
Neutrophil & $0.383(0.284-0.482$ & 4.75 & 0.026 & 44.9 & 43.8 & 32.8 & 56.5 \\
NLR & $0.357(0.259-0.455)$ & 2.22 & 0.007 & 42.9 & 40 & 30.4 & 53.3 \\
PLR & $0.386(0.285-0.488)$ & 114.6 & 0.031 & 48.9 & 42.5 & 34.3 & 57.6
\end{tabular}

As plAs plateleatelet MPV and lLeucosyte, were not significant, the cut-off values were not determined.AUC: Area under curve, $p: p$ value. PPV: Positive Predictive Value, NPV: Negative Predictive Value, NLR:Neutrophil/Lymphocyt, PLR: Platelet/Lymphocyte 


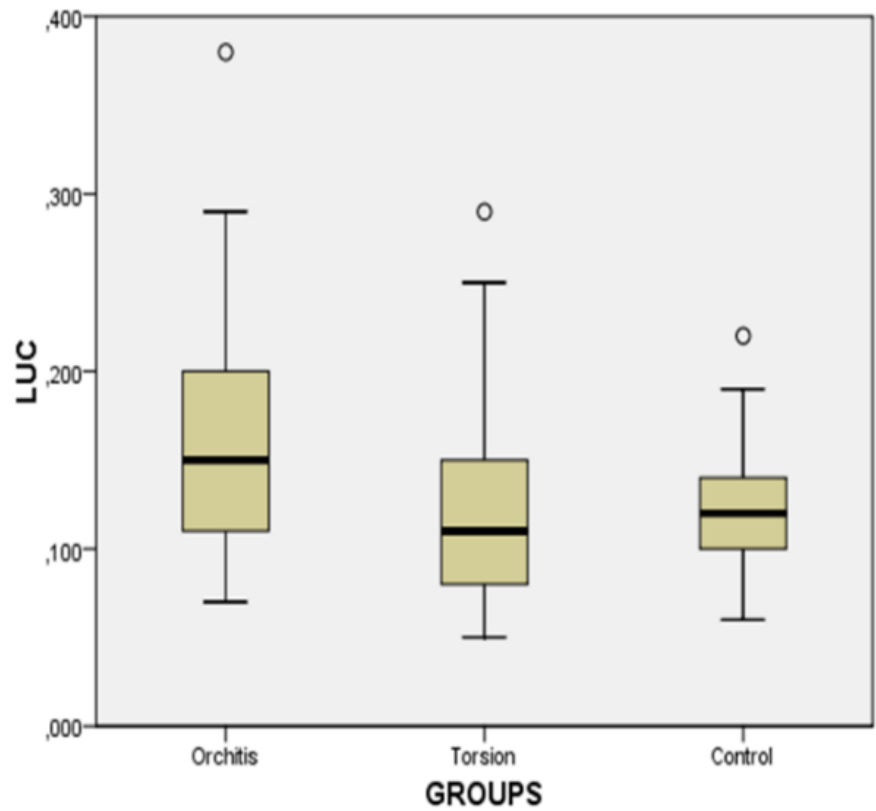

Figure 1. Box graph of large unstained cell (LUC) according to groups

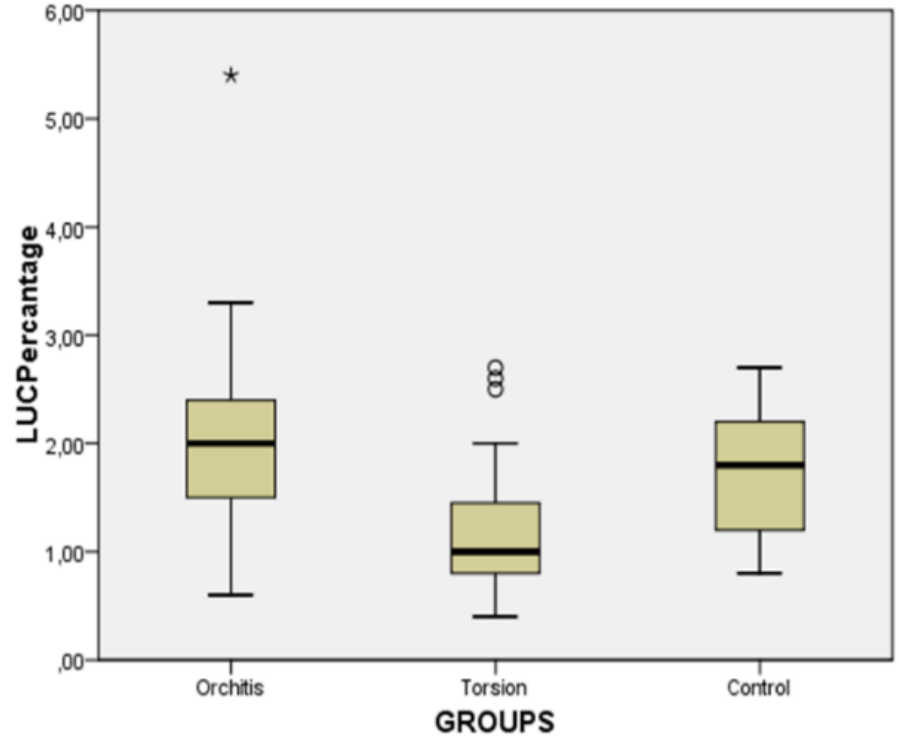

Figure 2. Box graph of large unstained cell (LUC) Percentage (\%) according to groups 


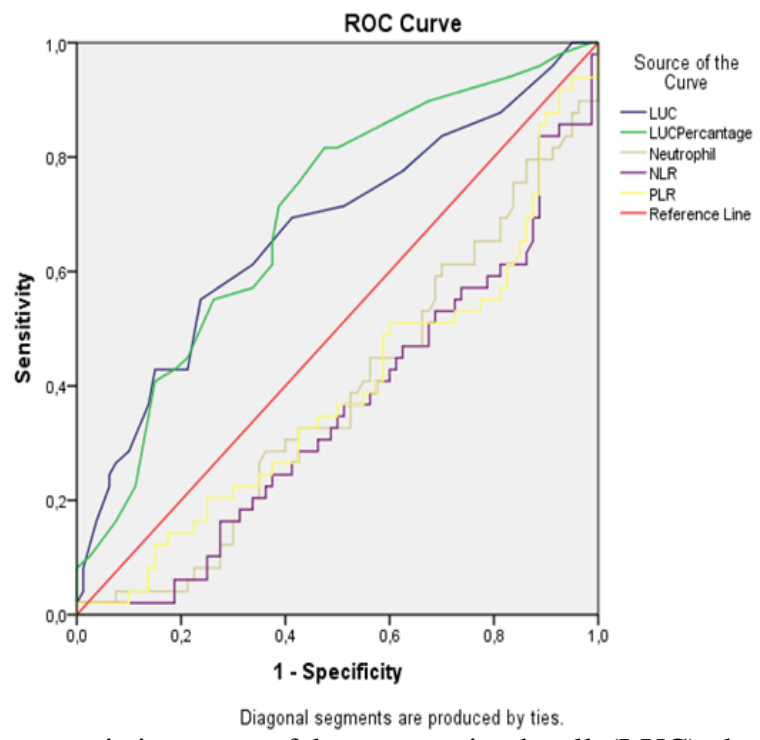

Figure 3. Receiver operating characteristic curve of large unstained cell (LUC), large unstained cell (LUC) \%, Neutrophil, Neutrophil/Lymphocyte (NLR) and Platelet/Lymphocyte(PLR) count

\section{Discussion}

The two most common causes of acute scrotum are TT and EO. TT is a urological emergency requiring emergency diagnosis and treatment as it can cause loss of the testis. The pathophysiology of these two diagnoses is different from each other. While the basic pathology is acute ischemia in torsion, it is an infective process in EO.

To date, many studies have been conducted with the idea that different hematological parameters could be used as biomarkers in the differential diagnosis of the two diseases. In a study of a paediatric age group, full blood count parameters such as erythrocyte sedimentation rate (ESR), C-reactive protein (CRP), and neutrophils were evaluated in the differential diagnosis of inflammatory and non-inflammatory diseases in the etiology of acute scrotum. A significant difference was found between the two groups especially in the neutrophil and CRP levels (8). In a study of procalcitonin to be used in the differential diagnosis of TT and EO, Yamis et al (9) determined elevated levels in EO. Conflicting results have been obtained in studies that have evaluated the utility of thrombocyte and MPV values in the differential diagnosis of EO and TT. Cicek et al found no difference between the two groups in the thrombocyte values, while Bitkin et al reported a significant difference between the thrombocyte counts of the two groups. The MPV values were found to be significant in both studies $(6,7)$. In the current study, these two biomarkers were not found to be statistically significant, which was thought to be for two reasons. The first was that the EDTA in the tubes used for the blood samples could have impaired the thrombocyte structure, and the second was that in literature, different results of both parameters have been shown to have been obtained in measurements made using different technologies (10).

It is also noticeable that there are different results in the literature related to the predictive values of leukocyte and neutrophil counts in the differential diagnosis of EO and TT. In a study the patient groups showed a significant elevation compared to the control group, but the predictive values were not found to be significantly high (10). In another study, the evaluation between groups was similar, but while the predictive value of leukocyte count was found to be statistically significant, the predictive value of the neutrophil count was not significant. Similar to the findings in the literature, when the role of the leukocyte count in differential diagnosis was evaluated in the current study, a significant difference was found between groups, but the predictive value was not determined to be significant.

Previous studies have examined the predictive value of NLR and PLR in the differential diagnosis of EO and TT. In all the studies on this subject in the literature, in both parameters, the difference between groups has been shown to be, significant and statistically significant predictive values have been determined. Similar results were obtained in the current study. However, the sensitivity and specificity values obtained as a result of the predictive evaluation were seen to be lower than those reported in the literature (Table 2).

Large unstained cells (LUC) are large peroxidase negative cells, which do not conform to other leukocyte subtypes, and these generally include 
virally activated lymphocytes, plasma cells, hairy cells, pediatric lymphocytes, and peroxidase negative lymphoblasts (11). The focus in the literature has been towards the possible use of LUC values as a marker for treatment response or prognosis particularly in hematological malignancies $(12,13)$. Moreover, it has been reported that they could be a marker in the differential diagnosis or prognosis of many infectious diseases (14-16). Therefore, this is the first study to have shown that LUC and LUC\% values can be used in the differential diagnosis of $\mathrm{EO}$ and TT. Both values were found to be significantly higher in the EO group than in the TT group (Table 1, Figure 1 and Figure 2). In addition, there was observed to be higher significance in all the other parameters examined in respect of predictive values (Figure 3, Table 2). This is not the first use of these values in differential diagnosis reported in literature, but this study is the first to have shown a comparison of a disease where the main pathology is ischemia and a disease where it is infection.

\section{Limitations}

Limitations of this study were that it was retrospective and conducted in a single centre. A further limitation was that parameters such as procalcitonin, CRP and Amyloid A, which are acute phase reactants, were excluded from the study as testing is expensive and they are not used routinely.

\section{Conclusions}

Complete blood count (CBC) is an inexpensive and rapidly accessible test, which can be easily accessed even in the most basic of healthcare centres. The parameters within CBC are of guidance for diseases that require comparison and a rapid decision. The differentiation of EO and TT encountered in the emergency polyclinic is a situation that requires emergency diagnosis and emergency treatment. Furthermore, scrotal Power Doppler USG, which should be used as a rule in the differential diagnosis, may not have the same reliability in every centre, as it is an experience-based examination and may not always be immediately available. The results of this study showed that the LUC and LUC\% values obtained from $\mathrm{CBC}$ can be reliably used in the differential diagnosis of EO and TT. Nevertheless, there is a need for further prospective multi-centre studies to strengthen the reliability of the use of these values and to enable them to be used routinely in emergency polyclinics.
Ethics Committee Approval: The protocol was approved by local Ethics Commitee at Ankara City Hospital (Protocol No: E1-20-1126).

Peer-review: Externally peer-reviewed.

\section{Author Contributions:}

Concept: B.B.K; Design: B.B.K, Literature Search: B.B.K, B.G, Data Collection and/or Processing: B.G, Analysis and/or Interpretation: B.B.K, B.G, Writing: B.B.K, B.G.

Conflict of Interest: No conflict of interest was declared by the authors.

Financial Disclosure: The authors declared that this study hasn't received no financial support.

\section{References}

1. Pogorelić Z, Mrklić I, Jurić I. Do not forget to include testicular torsion in differential diagnosis of lower acute abdominal pain in young males. $\mathrm{J}$ Pediatr Urol, 2013;9(6 PART B):1161-5.

2. Pogorelić Z, Mustapić K, Jukić M, Todorić J, Mrklić I, Meštrović J, et al. Management of acute scrotum in children: A 25-year single center experience on 558 pediatric patients. Can J Urol. 2016;23(6):8594-601.

3. Karakan T, Bagcyoglu M, Ozcan S, Telli O, Turgut H, Ozkan M, et al. Seasonal preponderance in testicular torsion: Is it a myth? Arch Esp Urol. 2015;68(10):750-4.

4. Mellick LB. Torsion of the Testicle. Pediatr Emerg Care. 2012;28(1):80-6.

5. Gunther P, Schenk JP, Wunsch R, Holland-Cunz $\mathrm{S}$, Kessler U, Troger J, et al. Acute testicular torsion in children: The role of sonography in the diagnostic workup. Eur Radiol 2006;16(11):2527-32.

6. Bitkin A, Aydın M, Ozgur BC, Irkilata L, Akgunes E, Keles M, et al. Can haematologic parameters be used for differential diagnosis of testicular torsion and epididymitis? Andrologia. 2018;50(1):1-6.

7. Cicek T, Togan T, Akbaba K, Narci H, Aygun C. The value of serum mean platelet volume in testicular torsion. J Int Med Res.2015;43(3):452_ 9.

8. Meštrović J, Biočić M, Pogorelić Z, Furlan D, Družijanić N, Todorić D, et al. Differentiation of inflammatory from non-inflammatory causes of acute scrotum using relatively simple laboratory tests: Prospective study. J Pediatr Urol [Internet]. 2013;9(3):313-7. 
9. Yamis S, Gedik A, Sahin H, Batun S, Nergiz Y, Bircan K. Is procalcitonin valuable in the differential diagnosis of testicular torsion and epididymo-orchitis. Saudi Med J. 2010;31(2):170-4.

10. Kartal O, Kartal AT. MPV: A reliable method to determine the prognosis? Vol. 37, Renal Failure. Informa Healthcare; 2015. p. 184.

11. Cakir I, Cakir N, Atalay MA, Koc AN. Large unstained cells are correlated with inflammatory biomarkers in patients with invasive aspergillosis. Turkish J Biochem. 2018;43(3):306-11.

12. Bononi A, Lanza F, Ferrari L, Gusella M, Gilli G, Abbasciano V, et al. Predictive value of hematological and phenotypical parameters on postchemotherapy leukocyte recovery. Cytom Part B - Clin Cytom. 2009;76(5):328-33.

13. Drewinko B, Bollinger $\mathrm{P}$, Brailas $\mathrm{C}$, Wyatt $\mathrm{J}$, Simson E, Trujillo JM. Flow cytochemical patterns of white blood cells in human haematopoietic malignancies. II. Chronic leukaemias. Br J Haematol. 1987;67(2):157-65.

14. Vanker N, Ipp H. Large unstained cells: A potentially valuable parameter in the assessment of immune activation levels in HIV infection. Acta Haematol 2014;131(4):208-12.

15. Bastug A, Bodur H, Erdogan S, Gokcinar D, Kazancioglu S, Kosovali BD, et al. Clinical and laboratory features of COVID-19: Predictors of severe prognosis. Int Immunopharmacol 2020; $1: 88$.

16. Shin D, Lee MS, Kim DY, Lee MG, Kim DS. Increased large unstained cells value in varicella patients: A valuable parameter to aid rapid diagnosis of varicella infection. J Dermatol. 2015;42(8):795-9. 